\title{
Proposal for a gel-based SERS sensor
}

\author{
$\operatorname{AUTHOR}(\mathrm{S})$ :
}

Samir, Kumar; Namura, Kyoko; Suzuki, Motofumi

\section{CITATION:}

Samir, Kumar ... [et al]. Proposal for a gel-based SERS sensor.

Proceedings of SPIE 2019, 10894: 1089414.

\section{ISSUE DATE: \\ 2019-06-07}

URL:

http://hdl.handle.net/2433/243209

\section{RIGHT:}

Copyright 2019 Society of Photo-Optical Instrumentation Engineers. One print or electronic copy may be made for personal use only. Systematic reproduction and distribution, duplication of any material in this paper for a fee or for commercial purposes, or modification of the content of the paper are prohibited. 


\section{Proposal for a gel-based SERS sensor}

\section{Samir Kumar, Kyoko Nomura, Motofumi Suzuki}

Samir Kumar, Kyoko Nomura, Motofumi Suzuki, "Proposal for a gel-based SERS sensor," Proc. SPIE 10894, Plasmonics in Biology and Medicine XVI, 1089414 (7 March 2019); doi: 10.1117/12.2506951

SPIE. Event: SPIE BiOS, 2019, San Francisco, California, United States 


\title{
Proposal for a gel-based SERS sensor
}

\author{
Samir Kumar*, Kyoko Namura, Motofumi Suzuki \\ Kyoto University, Department of Micro Engineering, Kyoto 615-8540, Japan
}

\begin{abstract}
The plasmonic nanostructures required for the SERS are commonly in the form of solid substrates, or as colloidal solutions, both of them are not very useful to detect the biomarkers directly on human skins. Gel-based SERS substrates, into which the plasmonic nanostructures are incorporated, will be helpful for the direct collection of the biomarkers from secretions such as sweat. To elucidate these points, we studied the diffusion of Raman probe 4, 4'-Bipyridine (BPY) in the cetyltrimethylammonium bromide:sodium salicylate (CTAB:NaSal) gel. Au nano-island SERS chip was coated with a 1:1 complex of CTAB:NaSal . Then, the diffusion of the probe was studied by SERS spectra as a function of time. The SERS signal intensity increases gradually with increasing time. Highly porous gel rapidly absorbed aqueous analyte solutions generating large SERS signals. The subsequent increase in signal could arise from the diffusion of the analyte molecule into the gel and onto the Au aggregates. Importantly, this gel-based SERS sensor did not significantly compromise the SERS performance of the analyte. We propose that this gel-based SERS sensor can be smeared directly onto the skin surface to absorb the body fluids from sweat, enabling the detection of biomarkers.
\end{abstract}

Keywords: SERS, hydrogel, sensors

\section{INTRODUCTION}

Recently, surface-enhanced Raman scattering (SERS) has emerged as an essential analytical tool for the applications ranging from detection of explosives, ${ }^{1}$ pesticide, ${ }^{2}$ bacteria ${ }^{3,4}$ and viruses ${ }^{5}$, non-invasive imaging for diagnostics to on-site sensing for disease bio-markers ${ }^{6-8}$. SERS substrates have been fabricated ranging from rough metal surfaces to fractals, nanowires and well-ordered substrates. ${ }^{9-12}$ The plasmonic nanostructures required for the SERS are commonly in the form of solid substrates (on glass or $\mathrm{Si}$ wafer) or as colloidal solutions. ${ }^{12,13}$ However, the solid substrates are rigid and brittle and hence, these static substrates severely limit the application of plasmonic nanostructures, such as to detect the biomarkers directly on human skin. The SERS substrate in colloidal solution may have very high sensitivity but lacks reproducibility. ${ }^{13}$ Plasmonic nanostructures incorporated gel-based SERS substrates are better suitable for the applications such as direct collection of the biomarkers from secretions (for instance sweat and urine). However, there are very few reports about the diffusion of molecules through the gel and on the study of an appropriate gel for the SERS applications. Doherty et al., demonstrated methylcellulose removable gels for investigating organic dyes and pigments in paint layers. ${ }^{14}$ Aime et al., reported the in situ growth of silver nanoparticles within gelatin gels and studied the (upto $100 \mathrm{~nm}$ ) thermoreversible optical shifts. ${ }^{15}$ Yao et al., ${ }^{16}$ and Shin et al. ${ }^{17}$ encapsulated Au nanoparticles inside the poly(vinyl alcohol) (PVA) and poly(acrylic acid) (PAA) hydrogel respectively. They reported that the gel encapsulated Au nanoparticles are stable for longer duration and reproducible. Lee et al., reported large thin, dry polymer sheets containing various SERS active Ag nanoparticles. ${ }^{18}$ They prepared these polymer sheets by drying an aqueous mixture of Hydroxyethylcellulose (HEC) and aggregated $\mathrm{Ag}$ colloid in $10 \times 10 \mathrm{~cm}$ molds. The primary purpose of their study was to protect the nanoparticle aggregates from the environment during storage and ease of handling. As per authors knowledge, no studies have been reported aimed at developing a gel-based SERS sensor for the direct collection of analyte from the body fluids and on the study of an appropriate gel for the SERS applications.

We propose a gel-based SERS substrate for the direct detection of biomarkers from the body fluids. Here we report a few preliminary results for the same. We coated Au nano-island SERS chip with a gel (1:1 complex of cetyltrimethylammonium bromide:sodium salicylate (CTAB:NaSal)). Then, we deposited $20 \mu \mathrm{L}$ droplets of $1 \mathrm{mM}$ aqueous BPYsolution onto the gel and recorded its SERS spectra as a function of time. Highly porous gel rapidly absorbed aqueous analyte solutions generating large SERS signals. The subsequent increase in signal could arise from the diffusion of the analyte molecule into the gel and onto the Au aggregates. Importantly, this gel-based SERS sensor did not significantly compromise the SERS performance of the analyte. We propose that this gel-based SERS sensor can be smeared directly onto the skin surface to absorb the body fluids from sweat, enabling the detection of biomarkers.

*samir.kumaprasr@okjestp-Bialodip and Medicine XVI, edited by Tuan Vo-Dinh, Ho-Pui A. Ho, Krishanu Ray, Proc. of SPIE Vol. 10894, 1089414 · C 2019 SPIE · CCC code: 1605-7422/19/\$18 · doi: 10.1117/12.2506951 


\section{METHODOLOGY}

Au nano-island SERS chip (Wavelet ${ }^{19}$ ) was purchased from Nidek Co., Ltd, Japan and coated was from the top with a gel (1:1 complex of CTAB:NaSal). Then, we deposited $20 \mu \mathrm{L}$ droplets of $1 \mathrm{mM}$ aqueous BPY solution onto the gel and recorded its SERS spectra as a function of time. SERS spectra were acquired using an inverted laser Raman Spectrometer (RAM150S) from the bottom of the SERS chip. The excitation source was a laser of $785 \mathrm{~nm}$, focused with a 10× objective.

\section{RESULTS AND DISCUSSION}

We studied the diffusion of the analyte through the gel. For that purpose, the Raman probe was dropped on top of the gel, and SERS spectra were recorded from the bottom. We compared the SERS signal collected from the top and bottom of the SERS chip to check for any loss in the scattering intensity. The SERS spectra of Raman probe BPY from top and bottom of the substrate is shown in Fig. 1. There is no significant loss in the SERS intensity when collected from the bottom side of the substrate.

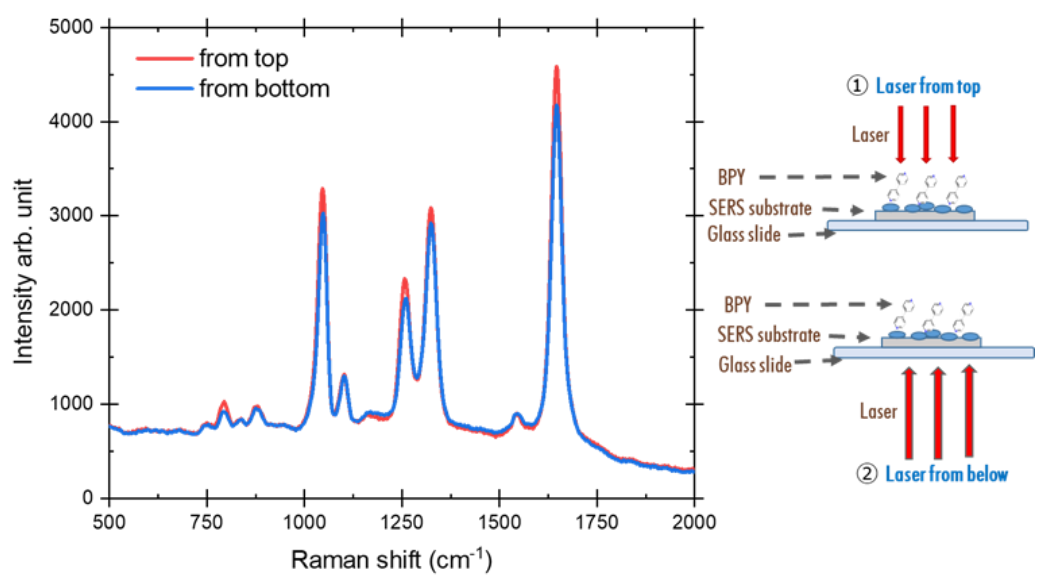

Figure 1. SERS spectra of Raman probe 4 4'-bipyridyl (BPY) from top and bottom of the substrate.

For the on-site diagnostic, we need to apply the gel onto the skin for collecting the biomarkers. For that purpose, the hydrogel should provide a good permeability through the gel network at the same time, the molecules comprising the gel matrix should display minimal inelastic scattering to provide unperturbed Raman spectra of the small molecules from the gel matrix. Fig. 2(a) shows the SERS spectra of gel only (black curve) and the Raman probe inside the gel. We observed no sharp and strong Raman background from the gel, and BPY SERS signal can be clearly distinguished from the gel background.

On applying the gel on the skin, the analyte (biomarkers) should be able to diffuse easily within the gel and reach the $\mathrm{Au}$ nanoparticles. To study the diffusion of the analyte inside the gel we dropped $20 \mu \mathrm{L}$ of BPY on top of the gel of different thicknesses $(\Delta t)$ and recorded the SERS spectra after certain time intervals from the bottom, Fig 2(b)-2(d). The characteristics peaks of BPY at $1085 \mathrm{~cm}^{-1}, 1240 \mathrm{~cm}^{-1}, 1509 \mathrm{~cm}^{-1}$ can be assigned to the A1 ring mode. The bands at 865 $\mathrm{cm}^{-1}$ could be assigned to the A2 mode. The B2 ring modes appeared at $1300 \mathrm{~cm}^{-1}, 1624 \mathrm{~cm}^{-1} \cdot{ }^{20,21}$ The diffusion time was found to depend upon the gel thickness. For $0.3 \mathrm{~mm}$ gel thickness, the intensity maximum intensity for the $1624 \mathrm{~cm}^{-1}$ was attained in about $30 \mathrm{~min}$. For $0.8 \mathrm{~mm}$ gel thickness, the intensity reached the maximum in about $45 \mathrm{~min}$. Hence, in a short duration, a significant amount of analyte can be collected using the gel. The maximum intensity for the case of $0.3 \mathrm{~mm}$ has higher counts than that of $0.8 \mathrm{~mm}$ and $2.8 \mathrm{~mm}$ thicknesses because of faster diffusion. Since the diffusion rate decreases with thickness $0.3 \mathrm{~mm}$ gel deliver a higher amount of analyte to the Au nano-islands. The time evolution of the $1624 \mathrm{~cm}^{-}$ ${ }^{1}$ peak is shown in Fig. 3. The SERS intensity first increased rapidly over time, eventually reaching a maximum at approximately $30 \mathrm{~min}$ (for $0.3 \mathrm{~mm}$ ), after which the signal started to decrease. For $0.3 \mathrm{~mm}$ thickness, the intensity falls 

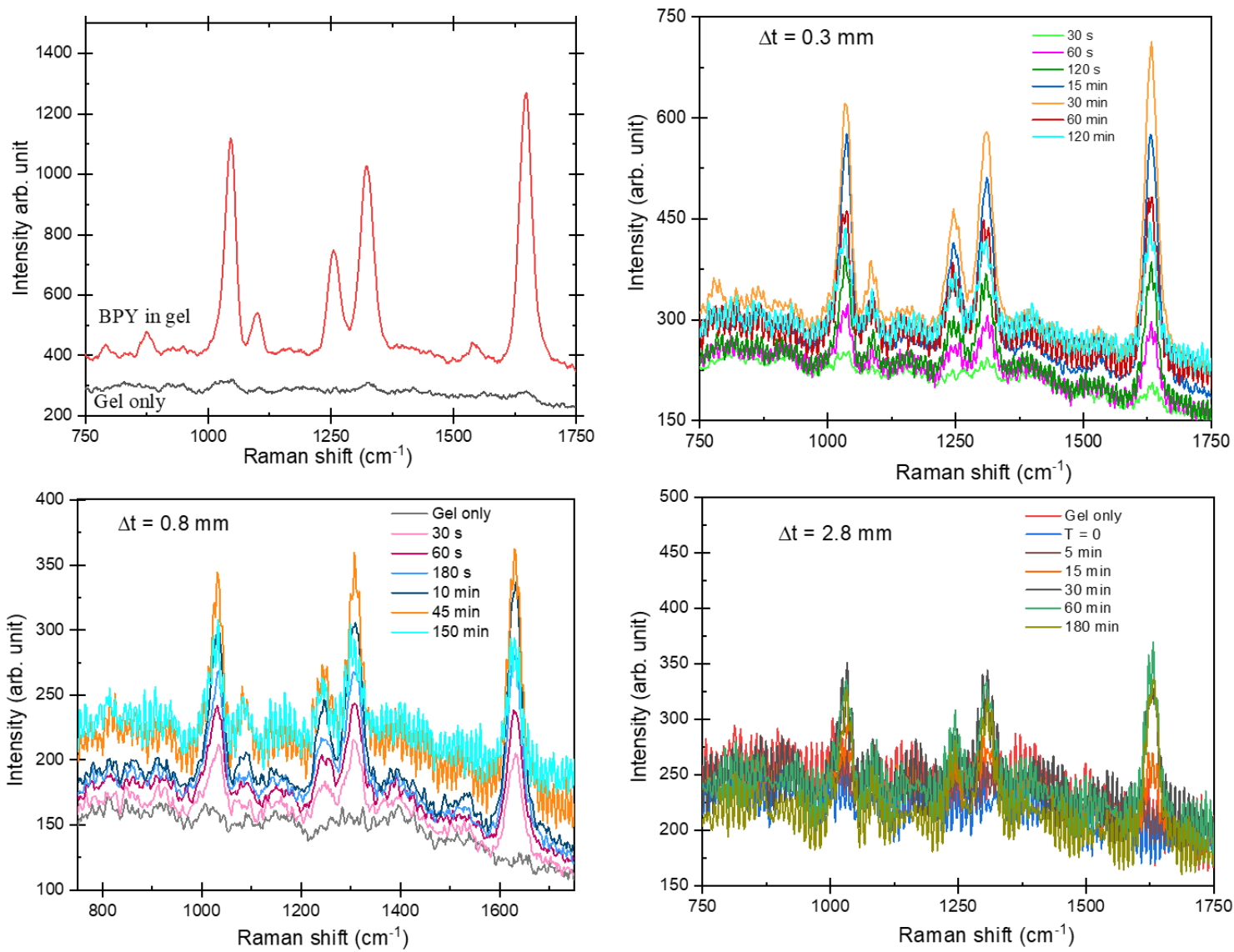

Figure 2. (a) Raman spectra of 1:1 complex of CTAB:NaSal gel (1\% v/v); (b) , (c) and (d) Time dependence of Raman spectra of BPY in gel of thickness $0.3 \mathrm{~mm}, 0.8 \mathrm{~mm}$ and $2.8 \mathrm{~mm}$ respectively.

rapidly (around $40 \%$ ) and attains plateau at approximately 70 min, after which the signal remained stable. For 0.8 and 2.8 $\mathrm{mm}$ thicknesses, the decrease in intensity is not very not very large (around 10\% and 5\% for 0.8 and $2.8 \mathrm{~mm}$ respectively). In general, the intensity reaches a plateau at $70 \mathrm{~min}$ for all the thicknesses.

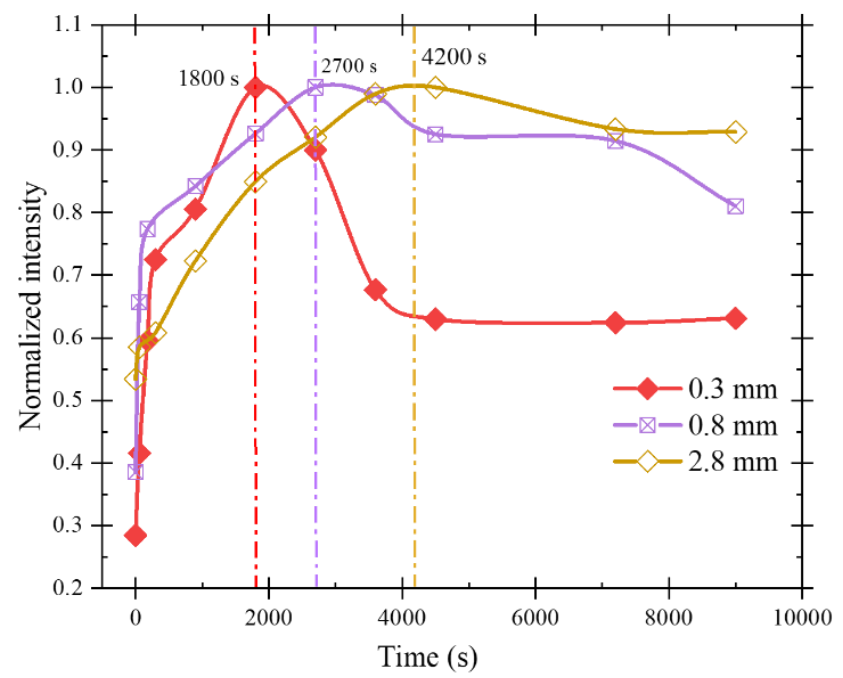

Figure 3. Time evolution of $1624 \mathrm{~cm}^{-1}$ peak of BPY for different thicknesses 
SERS is a surface phenomenon, and the monolayers of molecules within few $\mathrm{nm}$ of the metal nanoparticles contribute to the Raman signal enhancement. A large aggregation of analyte around the nanoparticles may also suppress the enhancement mechanism. ${ }^{14}$ Therefore, the reason behind the decrease in intensity for $0.3 \mathrm{~mm}$ thickness of gel may be the sudden avalanche of a large amount of analyte around the Au nano-islands that interferes with the SERS signal since the thickness is small. For the other thicknesses the diffusion process is slowed down due to the decrease in concentration with time before the analyte reaches the Au nano-island, hence does not interferes with the intensity. Therefore, by applying gel of thickness around $1 \mathrm{~mm}$ we can get good SERS intensity.

\section{CONCLUSIONS}

The CTAB:NaSal gel caused minimal perturbation and had a small Raman cross-section hence it does not interfere with signals from the analyte. The used gel provided good permeability and diffusion of the Raman probe through the gel. The diffusion time depends upon the gel thickness. It was demonstrated that Au nano-island gel composite could be a suitable candidate for the direct collection of the biomarker from body fluids.

\section{ACKNOWLEDGMENTS}

This work was supported by the Center of Innovation Program (COI) from the Japan Science and Technology Agency (JST) “The Last 5X Innovation R\&D Center for a Smart, Happy, and Resilient Society.”

\section{REFERENCES}

[1] Gillibert, R., Huang, J.Q., Zhang, Y., Fu, W.L., and Lamy de la Chapelle, M., "Explosive detection by Surface Enhanced Raman Scattering," TrAC Trends in Analytical Chemistry 105, 166-172 (2018).

[2] Kumar, S., Goel, P., and Singh, J.P., "Flexible and robust SERS active substrates for conformal rapid detection of pesticide residues from fruits," Sensors and Actuators B: Chemical 241, 577-583 (2017).

[3] Sengupta, A., Mujacic, M., and Davis, E.J., "Detection of bacteria by surface-enhanced Raman spectroscopy," Analytical and Bioanalytical Chemistry 386(5), 1379-1386 (2006).

[4] Kumar, S., K. Lodhi, D., Goel, P., Neeti, Mishra, P., and P. Singh, J., "A facile method for fabrication of buckled PDMS silver nanorod arrays as active 3D SERS cages for bacterial sensing," Chemical Communications 51(62), 12411-12414 (2015).

[5] Neng, J., Harpster, M.H., Wilson, W.C., and Johnson, P.A., "Surface-enhanced Raman scattering (SERS) detection of multiple viral antigens using magnetic capture of SERS-active nanoparticles," Biosensors and Bioelectronics 41, 316-321 (2013).

[6] Sinha, S.S., Jones, S., Pramanik, A., and Ray, P.C., "Nanoarchitecture Based SERS for Biomolecular Fingerprinting and Label-Free Disease Markers Diagnosis," Accounts of Chemical Research 49(12), 2725-2735 (2016).

[7] Ngo, H.T., Wang, H.-N., Burke, T., Ginsburg, G.S., and Vo-Dinh, T., "Multiplex detection of disease biomarkers using SERS molecular sentinel-on-chip," Analytical and Bioanalytical Chemistry 406(14), 3335-3344 (2014).

[8] Horstmeyer, R., Guenther, B., Kim, H.-J., and McCain, S., "Detection of Bilirubin using Raman Spectroscopy in a Neonatal Skull'2.

[9] Suzuki, M., Maekita, W., Wada, Y., Nagai, K., Nakajima, K., Kimura, K., Takao Fukuoka, and Mori, Y., “Ag nanorod arrays tailored for surface-enhanced Raman imaging in the near-infrared region," Nanotechnology 19(26), 265304 (2008).

[10] Bayata, F., Akinci, Z.B., Donatan, A.S., and Urgen, M., “A novel free-standing nanowire substrate with surface enhanced Raman scattering (SERS) activity," Materials Letters 67(1), 387-389 (2012).

[11] Rajput, A., Kumar, S., and Singh, J.P., "Vertically standing nanoporous Al-Ag zig-zag silver nanorod arrays for highly active SERS substrates,” Analyst 142(20), 3959-3966 (2017).

[12] Yang, L., Li, P., and Liu, J., "Progress in multifunctional surface-enhanced Raman scattering substrate for detection," RSC Advances 4(91), 49635-49646 (2014). 
[13] Sharma, B., Fernanda Cardinal, M., Kleinman, S.L., Greeneltch, N.G., Frontiera, R.R., Blaber, M.G., Schatz, G.C., and Van Duyne, R.P., "High-performance SERS substrates: Advances and challenges," MRS Bulletin 38(08), 615624 (2013).

[14] Doherty, M.D., Murphy, A., Pollard, R.J., and Dawson, P., "Surface-Enhanced Raman Scattering from Metallic Nanostructures: Bridging the Gap between the Near-Field and Far-Field Responses," Physical Review X 3(1), (2013).

[15] Aimé, C., Rietveld, I.B., and Coradin, T., "Hydrazine-induced thermo-reversible optical shifts in silver-gelatin bionanocomposites," Chemical Physics Letters 505(1-3), 37-41 (2011).

[16] Yao, S., Zhou, C., and Chen, D., "A highly porous PVA dried gel with gold nanoparticles embedded in the network as a stable and ultrasensitive SERS substrate," Chemical Communications 49(57), 6409 (2013).

[17] Shin, K., Ryu, K., Lee, H., Kim, K., Chung, H., and Sohn, D., “Au nanoparticle-encapsulated hydrogel substrates for robust and reproducible SERS measurement," The Analyst 138(3), 932-938 (2013).

[18] Lee, W.W.Y., Silverson, V.A.D., McCoy, C.P., Donnelly, R.F., and Bell, S.E.J., "Preaggregated Ag Nanoparticles in Dry Swellable Gel Films for Off-the-Shelf Surface-Enhanced Raman Spectroscopy," Analytical Chemistry 86(16), 8106-8113 (2014).

[19] Suzuki, M., Nakajima, K., Kimura, K., Fukuoka, T., and Mori, Y., “Au Nanorod Arrays Tailored for SurfaceEnhanced Raman Spectroscopy," Analytical Sciences 23(7), 829-833 (2007).

[20] Strekas, T.C., and Diamandopoulos, P.S., "Surface-enhanced raman spectroscopy of bipyridines and phenylpyridines," The Journal of Physical Chemistry 94(5), 1986-1991 (1990).

[21] Kurokawa, Y., Imai, Y., and Tamai, Y., "Surface-enhanced Raman Scattering Observations on Bipyridine, Phthalimide, Phenylethylamine and Theobromine by Using a Fine Silver Particle-doped Cellulose Gel Film," The Analyst 122(9), 941-944 (1997). 